ORIGINAL ARTICLE

\title{
Effects of leg massage on recovery from high intensity cycling exercise
}

\author{
A Robertson, J M Watt, S D R Galloway
}

Br J Sports Med 2004;38:173-176. doi: 10.1136/bjsm.2002.003186

See end of article for authors' affiliations .....................

Correspondence to: Dr Galloway, Department of Sports Studies, University of Stirling, Stirling FK9 4LA, Scotland, UK; s.d.r.galloway@ stir.ac.uk

Accepted 16 June 2003
Background: The effect of massage on recovery from high intensity exercise is debatable. Many studies on massage suffer from methodological flaws such as poor standardisation of previous exercise, lack of dietary control, and inappropriate massage duration.

Objective: To examine the effects of leg massage compared with passive recovery on lactate clearance, muscular power output, and fatigue characteristics after repeated high intensity cycling exercise, with the conditions before the intervention controlled and standardised.

Methods: Nine male games players participated. They attended the laboratory on two occasions one week apart and at the same time of day. Dietary intake and activity were replicated for the two preceding days on each occasion. After baseline measurement of heart rate and blood lactate concentration, subjects performed a standardised warm up on the cycle ergometer. This was followed by six standardised 30 second high intensity exercise bouts, interspersed with 30 seconds of active recovery. After five minutes of active recovery and either 20 minutes of leg massage or supine passive rest, subjects performed a second standardised warm up and a 30 second Wingate test. Capillary blood samples were drawn at intervals, and heart rate, peak power, mean power, and fatigue index were recorded.

Results: There were no significant differences in mean power during the initial high intensity exercise bouts $(p=0.92)$. No main effect of massage was observed on blood lactate concentration between trials $(p=$ $0.82)$ or heart rate $(p=0.81)$. There was no difference in the maximum power $(p=0.75)$ or mean power $(p=0.66)$ in the subsequent Wingate test, but a significantly lower fatigue index was observed in the massage trial $(p=0.04$; mean (SD) fatigue index $30.2(4.1) \%$ v $34.2(3.3) \%)$.

Conclusions: No measurable physiological effects of leg massage compared with passive recovery were observed on recovery from high intensity exercise, but the subsequent effect on fatigue index warrants further investigation.
$M$ assage research has produced equivocal findings in recent years. Some athletes and physiotherapists support claims that massage can aid recovery and optimise performance; however, most of the evidence is anecdotal. Research in the field of sports massage has been flawed by many methodological variations and poor experimental control during the test phase including: inconsistent massage duration, no standardisation of warm up, absence of a period of active recovery when comparing massage with other interventions, and often no standardisation of physical activity/work performed preceding the massage. The literature does, to some extent, support psychological benefits from massage, ${ }^{1}$ but physiological and performance benefits have never been consistently observed. ${ }^{2}$ Cafarelli and Flint ${ }^{3}$ suggested that, in a practical setting, massage could show performance improvements, but lack of control would devalue the results. Given that the beneficial effects of active recovery after intense exercise are well established, ${ }^{4-6}$ research on the effect of massage on recovery of muscle function should include active recovery of some sort in all phases of the experimental design. To date, only one study has adopted this type of design in an attempt to tease out any potential benefits of massage combined with active recovery versus active recovery alone or massage alone. ${ }^{7}$ These findings indicate a beneficial effect of a 15 minute combined intervention, compared with active recovery or massage alone, on performance in repeated $5 \mathrm{~km}$ cycling time trials (approximately 6.5 minutes of effort before and after the intervention). These data provide some evidence for the beneficial effects of massage when combined with a short active recovery process; however, the massage was short
(7.5 minutes), confined to the calf or hamstrings, and no indication of massage protocol or diet/activity control was provided. Furthermore, the main emphasis for an effect of massage in recovery from exercise is focused on improvements in blood flow and lactate clearance. ${ }^{3}$ Therefore it appears that there is a need for a controlled study incorporating certain aspects of a practical setting (inclusion of a short active recovery period), a more suitable length of massage (20-30 minutes), and greater experimental control (preceding diet and exercise), to assess the potential benefits of massage on lactate clearance and subsequent high intensity exercise capacity/performance. Furthermore, the literature on the role of massage in increasing blood flow or lactate clearance is equivocal, ${ }^{7-10}$ raising a question as to the precise role of massage in short term recovery. Therefore the aim of this work was to examine the effects of leg massage compared with passive recovery on lactate clearance, muscular power output, and fatigue characteristics after repeated high intensity cycling exercise, with the conditions before the intervention well controlled and standardised.

\section{METHODOLOGY}

After ethics committee approval, nine healthy male games players (aged 20-22 years) volunteered to participate in the study. All regularly took part in exercise involving bouts of high intensity work (rugby, football, and field hockey). Subjects entered the laboratory on three separate occasions one week apart and at the same time of day. Familiarisation was completed on the first visit to ensure that they all knew the protocol and could complete the amount of work required. After this familiarisation period, the subjects 
entered the experimental phase. Dietary intake (food and fluid) and exercise intensity and duration were recorded for two days before the familiarisation visit, and subjects then replicated this exactly in the two days before each subsequent laboratory visit. Subjects were instructed not to exercise heavily in the 24 hours before attending the laboratory for all visits. They were questioned about compliance with the dietary intake and exercise controls on arrival for each test session. They were then seated, and, after a 15 minute rest period, a baseline blood sample was drawn. Subjects then performed a standardised light warm up (consisting of five minutes of cycling at $80 \mathrm{~W}$ ) and a short stretching period (three minutes of static stretches of hamstrings, calf, and quadriceps muscle groups). They then completed six standardised 30 second high intensity bouts of exercise on a cycle ergometer, each interspersed with 30 seconds of active recovery $(40 \mathrm{~W})$. Power output was monitored during these high intensity bouts of exercise through a PC interface. On completion of the six high intensity bouts, subjects undertook five minutes of active recovery $(80 \mathrm{~W})$ and then a 20 minute intervention. The intervention was either 20 minutes of passive supine rest or 20 minutes of leg massage, assigned in a randomised cross over fashion. The massage was applied for five minutes to the back of the left leg followed by five minutes to the back of the right leg with the subject in a prone position on a standard treatment couch. The subject then assumed a supine position, and massage was applied to the front of the right then left leg (each for five minutes). Table 1 shows the massage protocol followed during each five minute period. Most strokes were grade 1 or 2, but three grade 3 effleurage strokes, using a clenched fist, were applied in a centripetal direction to the left and right iliotibial band midway through the supine massage. All massage was administered by the same chartered physiotherapist using a conventional bland mineral oil (40 ml contact medium was used per massage-that is, $10 \mathrm{ml}$ per massage area). After the intervention period, the subject then completed the same standardised five minute warm up and three minutes of static stretching as previously described, before a 30 second all out high intensity bout of exercise (Wingate test). Heart rate was recorded throughout the protocol, and capillary blood samples were drawn for lactate analysis at rest, after the six initial high intensity bouts, at 10 and 20 minutes of intervention, and three minutes after the Wingate test. Blood lactate was measured using a LM10 Little Champion analyser (Analox Instruments Ltd, London, UK). Wingate test variables were recorded through a PC interface and included peak power (W), mean power $(\mathrm{W})$, and fatigue index (percentage change in power output between the first five seconds and the last five seconds of the 30 second exercise period).

Statistical analysis was by repeated measures analysis of variance or one way analysis of variance as appropriate.

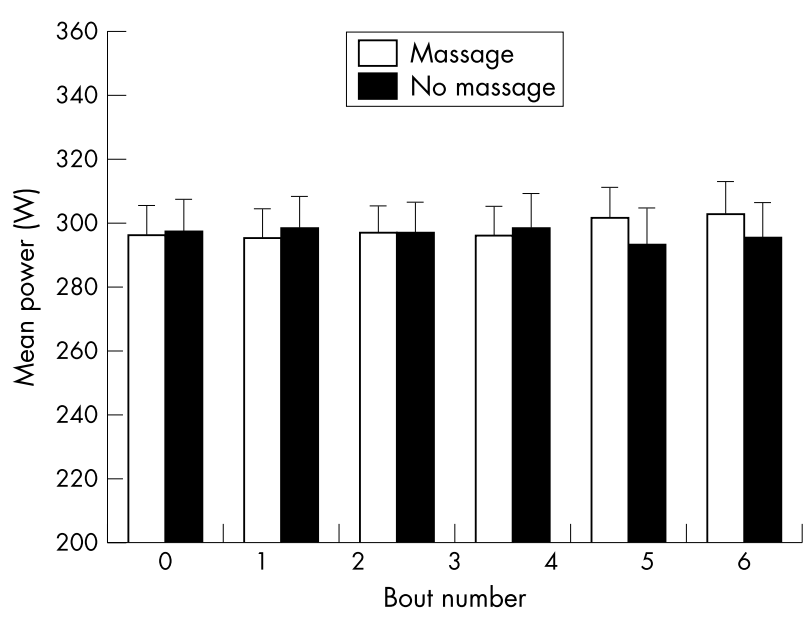

Figure 1 Mean (SEM) power output in each of the six initial high intensity exercise bouts. No significant difference was observed between trials at any time.

Significance was taken at $\mathrm{p}<0.05$. All values are reported as mean (SEM) in figures, mean (SD) in tables, with mean (95\% confidence interval (CI)) and effect size (ES) reported where appropriate. ES was calculated from the difference in mean values between groups divided by the pooled SD of the data for both groups. An ES of less than 0.2 was considered a small difference, and an ES of greater than 0.8 was considered a large difference. ${ }^{11}$

\section{RESULTS}

All subjects coped well with the test procedures and all indicated compliance with pretest diet and exercise controls. There was no significant difference between trials in the power output sustained during the initial standardised high intensity exercise bouts ( $p=0.92$; fig 1 ). Blood lactate concentration was not significantly different between trials at any time point ( $\mathrm{p}=0.82$; fig 2 ). No significant difference in heart rate response was noted at any time before, during, or after the intervention ( $p=0.81$, table 2 ). Performance in the high intensity exercise bout after the intervention (Wingate test) was not significantly different between trials (fig 3) with no change in corrected peak power $(\mathrm{p}=0.75)$ or corrected mean power $(\mathrm{p}=0.66)$. The mean $(95 \% \mathrm{CI})$ difference between massage and passive rest trials was -20.3 $(-77.5$ to 37.0$)$ for peak power $(\mathrm{W})$, and 13.7 ( -31.1 to 58.5$)$ for mean power $(\mathrm{W})$. The calculated ES values were 0.16 and 0.22 for peak and mean power respectively. A significant effect was observed on the fatigue index $(p=0.04)$. Mean (95\% CI) fatigue index (\%) was $30.2(27.5$ to 32.9$)$ in the massage intervention trial and 34.2 (32.1 to 36.3) in the passive intervention trial (fig 3). The mean (95\% CI)

\begin{tabular}{lll} 
Table 1 & Massage protocol & \\
\hline $\begin{array}{l}\text { Massage } \\
\text { technique }\end{array}$ & Description & Grade \\
\hline Stroking & Whole hand two handed in a centripetal direction & 4 strokes grade 1, two strokes \\
& grade 2 \\
Effleurage & Whole hand two handed, centripetal and multidirectional & Grade 1 up to grade 2 \\
Kneading & Whole hand two handed, centripetal and centrifugal & Grade 1 up to grade 2 \\
Picking up & Whole hand two handed v-shaped, centripetal & Grade 1 up to grade 2 \\
Wringing & Whole hand two handed, centripetal, centrifugal, multidirectional & Grade 1 \\
Rolling & Muscle rolling, centripetal & Grade 2 \\
Effleurage & Whole hand two handed, centripetal & Grade 2 \\
\hline
\end{tabular}

The protocol represents the procedures followed during each of the four five minute massage periods. All petrissage was interspersed with effleurage grade 2 in a centripetal direction. 


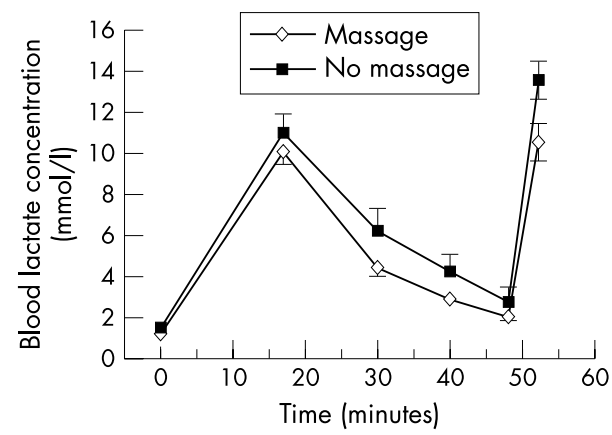

Figure 2 Mean (SEM) blood lactate concentration at rest, after the initial high intensity exercise bouts, at 10 and 20 minutes of intervention, immediately before the Wingate test, and three minutes after the Wingate test. No significant difference was observed between trials at any time.

difference in fatigue index between massage and passive rest trials was $-4.0(-6.6$ to -1.4$)$. The ES value for the fatigue index data was 0.98 . Table 3 shows mean values and pooled SD for Wingate test variables.

\section{DISCUSSION}

Under the well controlled and standardised conditions in this study, no measurable effect of leg massage was observed on lactate clearance after repeated bouts of high intensity effort. However, the significant difference in fatigue profile in the Wingate test indicates a slight difference in muscular performance related to a non-significant lower peak power and non-significant greater mean power in the massage intervention trial. No difference in blood lactate concentration was observed across the intervention period with passive recovery or massage, when both interventions were preceded by a short (five minute) active recovery period. The lack of an observed effect on lactate clearance with massage compared with passive rest implies that there was no change in muscle blood flow and/or lactate efflux during the massage intervention or that lactate removal from the circulation was unaffected by massage. These findings are consistent with a growing number of studies in this area. ${ }^{8} 1012$

One of the main concerns raised about previous studies on massage is methodological problems. ${ }^{13}$ Some of the main criticisms can be aimed at standardisation of previous exercise, length and type of massage intervention, and poor experimental control. In this study, the exercise workload and cadence were controlled to ensure that the total amount of work performed in the exercise task before the intervention was the same on both trials. The length and type of massage is often scrutinised in massage studies, and it has been recommended on a practical level that leg massage should last for about 10 minutes, with most massages requiring 10-30 minutes for effectiveness. ${ }^{14}$ Furthermore, with physiotherapists being largely responsible for massage provision at major athletics events, and with a ratio of one
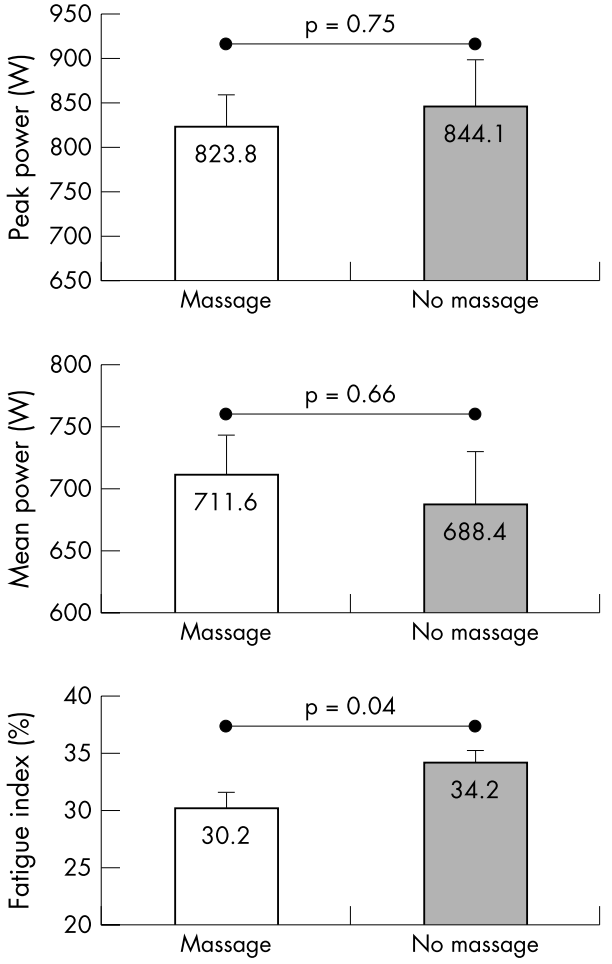

Figure 3 Mean (SEM) corrected peak power, corrected mean power, and fatigue index in the Wingate test after the intervention. No significant difference was observed between trials for peak or mean power, but a significant difference $(p=0.04)$ was noted for fatigue index.

physiotherapist for every 20 athletes at these events, the duration of massage practically possible is 20-30 minutes. The type of massage delivered depends on the aim of the intervention: in this study, we aimed to complete a leg massage that would commonly be used during recovery periods in track and field events, therefore the type and intensity of strokes were selected based on this aim. A crucial failure in many previous studies is a lack of detailed information on the type, intensity, and duration of strokes used in the massage. ${ }^{72}$ Without this information, the appropriateness of the intervention cannot be evaluated, and more importantly it cannot be reproduced. Many other methodological control problems are apparent in massage literature, including failure to standardise exercise and dietary intake in the days preceding the visits to the laboratory, or at least failure to report this information. ${ }^{7}$ Without this information, the reader is unable to ascertain whether muscle glycogen content was the same at the start of exercise, and whether acid-base status was likely to be similar; both of these factors could significantly affect the ability to perform high intensity exercise and the metabolite responses to exercise and massage. ${ }^{15-17}$ In our work, the

Table 2 Mean (SD) heart rate response (beats/min) at rest just before the start of warm up, and at the end of each of the following stages: initial warm up, standardised high intensity exercise bouts, five minutes of active recovery, 20 minute intervention, final warm up, and Wingate test in the massage and no massage trials

\begin{tabular}{llllllll}
\hline & Rest & Initial warm up & $\begin{array}{l}\text { High intensity } \\
\text { bouts }\end{array}$ & Active recovery & Intervention & Final warm up & Maximum (Wingate) \\
\hline Massage & $86(4)$ & $116(10)$ & $174(8)$ & $133(11)$ & $77(15)$ & $127(10)$ & $182(8)$ \\
No massage & $93(6)$ & $115(11)$ & $176(11)$ & $135(15)$ & $77(11)$ & $128(7)$ & $183(7)$ \\
\hline
\end{tabular}




\begin{tabular}{|c|c|c|c|c|c|}
\hline Variable & Massage trial & $\begin{array}{l}\text { Passive rest } \\
\text { trial }\end{array}$ & Pooled SD & Effect size & $\begin{array}{l}\% \text { change in } \\
\text { value between } \\
\text { trials }\end{array}$ \\
\hline Maximal power (W) & 823.8 & 844.1 & 130.5 & 0.16 & 2.4 \\
\hline Average power (W) & 711.6 & 688.4 & 107.7 & 0.22 & 3.4 \\
\hline Fatigue index (\%) & 30.2 & 34.2 & 4.1 & 0.98 & 11.7 \\
\hline
\end{tabular}

\section{Take home message}

This work adds to a body of literature that does not support the use of massage in recovery from exercise where the aim is to enhance lactate clearance. However, a positive effect of massage on fatigue index was observed in a subsequent high intensity cycle sprint test, which may reflect a change in muscle function. The effects of leg massage on muscle force production and fatigue characteristics need to be clarified.

control of dietary intake and activity should have ensured that pre-exercise muscle glycogen content and acid-base balance were the same before the intervention. Therefore, any observations made should be the direct result of the intervention rather than extraneous factors. It is interesting to note that, in this study, which used a similar protocol to that of Monedero and Donne, ${ }^{7}$ we observed that massage after active recovery of short duration resulted in an improved fatigue profile in a subsequent high intensity exercise task. These combined observations may indicate improved fatigue resistance after massage, which warrants further investigation.

The effectiveness of massage in the recovery of muscle function after fatiguing exercise has been the subject of several reviews, ${ }^{2}{ }^{18}$ and no clear evidence for a beneficial physiological effect has been observed. However, some of the literature on massage and stretching of the muscles before exercise indicates that there could be detrimental effects on maximal muscular power ${ }^{19} 20$ and/or muscle force production, ${ }^{21} 22$ which appears to contradict the findings of Monedero and Donne. ${ }^{7}$ Fowles et $a l^{21}$ examined the effects of 30 minutes of maximal passive stretching on force loss in human skeletal muscle and observed a $28 \%$ decline in voluntary force production immediately after the stretch period. Thereafter voluntary force production improved, but was still significantly lower at 60 minutes than before the stretching. The early force loss (up to 15 minutes after the stretching) was explained by reduced neural activation of the muscle as well as reduced muscle force-generating capacity (linked to reduced muscle stiffness). In this study, the Wingate test was conducted eight minutes after the intervention. With this in mind, the observed reduction in fatigue index (improved fatigue profile) after massage may be related to a small negative effect of massage and stretching on muscle activation, muscle stiffness, and force-generating capacity. These small effects may have affected the initial power output attainable in the Wingate test. Indeed, maximum power was slightly lower on the massage than the passive rest trial, and this translated into slightly higher mean power output and thus lower fatigue index. This speculation warrants further investigation such as identification of the size and duration of this effect so that practical guidelines can be prepared for strength/power athletes.

In conclusion, we did not observe any measurable difference in the effect of leg massage and supine passive rest on recovery of blood lactate after high intensity exercise. We cannot therefore support a hypothesis of improved lactate clearance with massage compared with passive rest after a short active recovery period. However, the data do reveal an effect of massage on the fatigue index in the Wingate test, which indicates a difference in test profile/muscle function that warrants further investigation.

\section{Authors' affiliations}

A Robertson, S D R Galloway, Department of Sports Studies, University of Stirling, Stirling FK9 4LA, Scotland, UK

J M Watt, Department of Sports Studies and University Physiotherapy Clinic, Stirling

\section{REFERENCES}

1 Hemmings B, Smith M, Graydon J, et al. Effects of massage on physiological restoration, perceived recovery, and repeated sports performance. Br J Sports Med 2000;34:109-15.

2 Tiidus PM. Manual massage and recovery of muscle function following exercise: a literature review. J Orthop Sports Phys Ther 1997;25:107-12.

3 Caferelli $E$, Flint $F$. The role of massage in preparation for and recovery from exercise: an overview. Sports Med 1992;14:1-9.

4 Ahmaidi S, Granier P, Taoutaou Z, et al. Effects of active recovery on plasma lactate and anaerobic power following repeated intensive exercise. Med Sci Sports Exerc 1996;28:450-6.

5 Dodd S, Powers SK, Callender T, et al. Blood lactate disappearance and various intensities of recovery exercise. J Appl Physiol 1984;57:1462-5.

6 Weltman A, Stamford BA, Fulco C. Recovery from maximal effort exercise: lactate disappearance and subsequent performance. J Appl Physiol 1979:47:677-82.

7 Monedero J, Donne B. Effect of recovery interventions on lactate removal and subsequent performance. Int J Sports Med 2000;21:593-7.

8 Martin NA, Zoeller RF, Robertson RJ, et al. The comparative effects of sports massage, active recovery, and rest in promoting blood lactate clearance after supramaximal leg exercise. Journal of Athletic Training 1998;33:30-5.

9 Shoemaker JK, Tiidus PM, Mader R. Failure of manual massage to alter limb blood flow: measures by Doppler ultrasound. Med Sci Sports Exerc 1997;29:610-14.

10 Dolgener FA, Morien A. The effect of massage on lactate disappearance. J Strength Condit Res 1993;7:159-62.

11 Vincent WJ. Statistics in kinesiology. 2nd ed. Champaign, IL: Human Kinetics, 1999.

12 Gupta S, Goswami A, Sadhukhan AK, et al. Comparative study of lactate removal in short term massage of extremities, active recovery and a passive recovery period after supramaximal exercise sessions. Int J Sports Med 1996;17:106-10.

13 Brown Menard M. Methodological issues in the design and conduct of massage therapy research. In: Jewell Rich G, ed. Massage therapy: the evidence for practice. London: Mosby, 2002:27-41.

14 Watt J. Massage for sport. Marlborough: Crowood Press, 1999.

15 Jones NL, Sutton JR, Taylor R, et al. Effects of $\mathrm{pH}$ on cardiorespiratory and metabolic responses to exercise. J Appl Physiol 1977:43:959-64.

16 Sutton JR, Jones NL, Toews CJ. Effect of $\mathrm{pH}$ on muscle glycolysis during exercise. Clin Sci 1981;61:331-8.

17 Greenhaff PL, Gleeson M, Maughan RJ. The effects of dietary manipulation on blood acid-base status and the performance of high-intensity exercise. Eur J Appl Physiol 1987;56:331-7.

18 Ernst E. Does post-exercise massage treatment reduce delayed onset muscle soreness? A systematic review. Br J Sports Med 1998;32:212-14.

19 Goodwin JE. A comparison of massage and sub-maximal exercise as warmup protocols combined with a stretch for vertical jump performance. J Sports Sci 2002; 20:48-9.

20 Cornwell A, Nelson AG, Sidaway B. Acute effects of stretching on the neuromechanical properties of the triceps surae muscle complex. Eur J Appl Physiol 2002;86:428-34.

21 Fowles JR, Sale DG, MacDougall JD. Reduced strength after passive stretch of the human plantarflexors. J Appl Physiol 2000;89:1179-88.

22 Behm DG, Button DC, Butt JC. Factors affecting force loss with prolonged stretching. Can J Appl Physiol 2001;26:261-72. 